\title{
Cardiomyocyte marker expression in dogs with left atrial enlargement due to dilated cardiomyopathy or myxomatous mitral valve disease
}

\author{
Izabela Janus ${ }^{1}$, Malgorzata Kandefer-Gola ${ }^{1}$, Rafal Ciaputa ${ }^{1}$, Agnieszka Noszczyk-Nowak ${ }^{2}$, \\ Urszula Paslawska ${ }^{2}$, Massimiliano Tursi ${ }^{3}$, Marcin Nowak ${ }^{1}$
}

${ }^{1}$ Division of Pathomorphology and Veterinary Forensics, Department of Pathology, Wroclaw University of Environmental and Life Sciences, Wroclaw, Poland

${ }^{2}$ Department of Internal Medicine and Clinic of Diseases of Horses, Dogs and Cats, Wroclaw University of Environmental and Life Sciences, Wroclaw, Poland

${ }^{3}$ Department of Animal Sciences, University of Turin, Grugliasco (TO), Italy

\begin{abstract}
Introduction. Dilated cardiomyopathy (DCM) and myxomatous mitral valve disease (MMVD) are common heart conditions in dogs. They have different etiology and pathogenesis and although other studies focused on changes in the left ventricles of the affected hearts, the aim of our study was to assess the expressions of some intrinsic proteins in the enlarged left atria.

Material and methods. We performed an immunohistochemical analysis of left atrial specimens obtained from 15 dogs with DCM, 35 dogs with MMVD and six control dogs. We assessed the expression of following proteins: SERCA1, SERCA2, sarcomeric actinin, smooth muscle actin, and dystrophin.

Results. We noted a higher percentage of SERCA1-positive cells in the MMVD group and lower percentage of dystrophin-positive cells in the DCM group as compared to control group. The expression of other proteins was similar in the hearts of control dogs and dogs with heart diseases.

Conclusions. The observed changes in the expression patterns of some proteins in the atria of dogs with DCM and MMVD suggest that atrial enlargement relies not only on volume overload, but also alterations of the intrinsic proteins can contribute to the pathogenesis of dilated cardiomyopathy. (Folia Histochemica et Cytobiologica 2017, Vol. 55, No. 2, 52-61)
\end{abstract}

Key words: dog; heart failure; dilated cardiomyopathy; myxomatous mitral valve disease; left atrium; dystrophin; SERCA1; IHC

\section{Introduction}

Dilated cardiomyopathy (DCM) and myxomatous mitral valve disease (MMVD) are the most common cardiac disorders in large (DCM) or small (MMVD) breed dogs. They are both accompanied by heart

\footnotetext{
Correspondence address: I. Janus, MVSc

Department of Pathology

Wroclaw University of Environmental and Life Sciences

C.K. Norwida 31, 50-375 Wroclaw, Poland

tel./fax: +48 713205415

e-mail: izabela.janus@upwr.edu.pl
}

enlargement, including mostly left ventricle and left atrium, although the pathomechanisms of these changes seem to vary between both diseases [1-5]. MMVD involves a progressing degeneration of mitral valve leaflets, which results in an increased mitral regurgitation blood flow. These changes directly lead to heart remodeling which particularly involves the left atrium [5]. The mechanism of left ventricular and left atrial enlargement in DCM is complex and probably does not result mainly from the chamber volume overload, although has not yet been well defined. Various factors like structural protein defects or impaired tissue oxygen supply are suspected to play 
a role in this process [4]. The majority of studies on heart failure resulting from these diseases focuses on changes occurring in the heart ventricles. However, current research suggests that myocardial remodeling occurs not only in the ventricle, but may be even more pronounced in atrial tissue [6-7].

During heart failure various myocardial proteins (including proteins of cardiomyocyte and extracellular matrix) present altered expression in heart muscle [8-11]. Atrial tissue undergoes different remodeling, as compared to ventricular cardiac muscle in various heart diseases, including DCM and congestive heart failure associated with arrhythmia $[6,12,13]$.

Our previous study [11] showed that in dogs with congestive heart failure and left atrial enlargement various myocardial proteins undergo changes in their expression in left atrial myocardium. Based on that preliminary study we decided to further explore the alterations in the left atrial cardiomyocytes during heart failure due to DCM and MMVD, including the expression of sarcoplasmic reticulum $\mathrm{Ca}^{2+}$ ATPase 2A-1 (SERCA1) protein, sarcoplasmic reticulum $\mathrm{Ca}^{2+}$ ATPase 2A-2 (SERCA2) protein, sarcomeric alpha-actinin (SA), alpha-smooth muscle actin (SMA), and dystrophin (DMD).

Sarcoplasmic reticulum $\mathrm{Ca}^{2+}$ ATPases are responsible for maintaining a sufficient cytosolic $\mathrm{Ca}^{2+}$ concentration during muscle contraction-relaxation cycles [14,15]. SERCA2 is the major isoform expressed in adult mammalian heart with higher protein level in atria as compared to the ventricles $[14,15]$. The chamber-related changes in protein expression may be connected with shorter duration of cardiomyocytes' contraction in atria than in ventricles [14]. The decrease in SERCA2 levels is associated with impaired myocardial function [14, 15]. SERCA1 is the isoform expressed mainly in skeletal muscles. However, there is evidence that its expression is altered in failing myocardium in humans and animals, changing the rate of $\mathrm{Ca}^{2+}$ transport $[15,16]$. The changes in SERCA expression might be a part of a natural adaptive process in the failing heart [16].

Cardiac alpha actin and skeletal muscle alpha actin are the major sarcomeric actin isoforms detected in striated muscles [17]. Smooth muscle actin produces a strong cytoplasmic staining in most smooth muscle cells, including those that form blood vessels [18]. In normal cardiac tissue the staining for SMA is restricted to smooth muscle cells of the vascular walls, whereas the cardiomyocytes are clearly negative [18]. During heart failure the amount of SMA-positive cells increases [19].

Dystrophin belongs to a group of membrane-associated proteins. Along with spectrin, talin, vinculin and ankyrin, it is involved in the fixation of sarcomeres to sarcolemma and stabilization of the plasma membrane $[8,20]$. A dystrophin missense mutation may cause X-linked dilated cardiomyopathy in both humans and dogs. In the disease, the heart muscle is mechanically weak and the contraction of the cell leads to membrane damage, muscle necrosis and degeneration [8, 20-22]. Apart from being a cause of muscle diseases, DMD was also found to be down-regulated in consequence of myocardial injury and ischemia of various origins [23, 24].

The aim of the study was to determine the expression of SERCA1, SERCA2, SA, SMA, and DMD proteins in left atrial tissue of dogs with dilated cardiomyopathy or myxomatous mitral valve disease.

\section{Material and methods}

Population study and inclusion criteria. The study was conducted on left atrial specimens obtained from 56 dogs divided into 3 groups: dogs presenting with dilated cardiomyopathy (DCM group; $\mathrm{n}=15)$, dogs with left atrial enlargement due to myxomatous mitral valve disease (MMVD group; $\mathrm{n}=35$ ) and dogs that underwent euthanasia due to causes not related to cardiovascular system and that did not show intravital or post-mortem features of cardiorespiratory disease and left atrial (LA) enlargement (control group; $\mathrm{n}=6$ ).

Dogs met the inclusion criteria if they had an enlarged left atrium as defined by an echocardiographic examination (left atrial (LA)-to-aortic root (Ao) diameter ratio; LA/ /Ao > 1.7 [1]), which was confirmed by the post-mortem examination. A dilation of the left ventricle and a decreased fractional shortening ( $\mathrm{FS}<20 \%$ ), both noted during the echocardiographic examination $[2,4,25]$, with normal or type 1 mitral valve lesions noted in autopsy (according to Whitney [26]) were required to classify dogs in the DCM group. Dogs in the MMVD group were required to have a preserved left ventricular systolic function (FS $>20 \%$ as revealed by echocardiographic examination) with type 3 or 4 mitral valve lesions (according to Whitney [26], found during autopsy). At the time of death or euthanasia, all the dogs in the DCM and MMVD groups presented with stage D heart failure (according to the ACVIM Consensus Statement [27]).

Clinical and post-mortem examination. Forty dogs underwent a standard clinical and cardiological examination with ECG, an echocardiographic examination and 24-hour ECG (Holter) monitoring in cases where rhythm disturbances were suspected as described in our previous study [11]. After the examination, dogs in the DCM and the MMVD group underwent appropriate treatment to reduce the effects of progressive heart failure and/or arrhythmias, and were checked every 6 months or more frequently if necessary 
until the moment of death or euthanasia. All the dogs in the DCM and the MMVD group died or were euthanized within five years of diagnosis.

In 16 cases, some or all the results of the cardiac examination were not available. In those cases, a particular emphasis was put on the post-mortem examination to confirm the inclusion of the dogs in the DCM, MMVD, or control group.

The results of the echocardiographic examination (left atrial and left ventricular enlargement) were confirmed during the autopsy, and the type of mitral valve lesion was assessed. Heart measurements were taken using a manual $150 \mathrm{~mm}$ Beerendonk caliper, accurate to the nearest $1 / 20$ $\mathrm{mm}$. The measurements were taken in planes compatible with those used during the echocardiographic examination. The left atrial diameter was related to the diameter of the aorta (as in LA/Ao ratio) and the left ventricular internal diameter and wall thickness were measured beneath the mitral valve between the papillary muscles. The type of mitral valve lesion according to Whitney [26] was determined based on the thickness of the valve leaflets and tendinous chords. Specimens from the left atrial wall were collected from each dog for further histopathological and immunohistochemical analyses. The specimens were collected in the dorso-ventral plane from the middle portion of the atrial free wall, at the same site in all the dogs.

Histopathological examination. The specimens were fixed in $7 \%$ buffered formalin, dehydrated, embedded in paraffin blocks and sectioned at $4 \mu \mathrm{m}$. Each specimen was randomly assigned a number to allow a blinded examination. The specimens were stained with hematoxylin-eosin or Masson-Goldner trichrome methods and evaluated for interstitial and perivascular fibrosis, cardiomyocyte degeneration (including cardiomyocyte nuclei enlargement, loss of cross striation and altered cardiomyocyte structure), intramyocardial arterial narrowing and inflammatory infiltrates as described in our previous studies $[11,12]$. Each of the features was evaluated in 20 randomly chosen fields per slide (for interstitial fibrosis, cardiomyocyte degeneration and inflammatory infiltrates) or in at least 10 vessels per slide (for perivascular fibrosis and intramyocardial arterial narrowing). An average score was counted for each feature in every specimen as described previously [11, 12].

Immunohistochemical examination. The paraffin sections were placed on silanized microscopic slides and underwent a standardized immunohistochemical (IHC) staining procedure. The sections were overlaid with primary antibodies, including a mouse monoclonal anti-ATP2A-1 - clone 4B8 (dilution 1:600, SERCA1 protein; Sigma-Aldrich, St. Louis, MO, USA), a rabbit polyclonal anti-ATP2A-2 (dilution 1:800, SERCA2 protein; Sigma-Aldrich), a mouse monoclonal anti-alpha-actinin (sarcomeric) — clone EA-53 (dilution 1:500, specific for both alpha-skeletal muscle and alpha-car- diac muscle actinin Sigma-Aldrich), a mouse monoclonal anti-alpha-smooth muscle actin — clone 1A4 (Sigma-Aldrich; dilution 1:1000); a rabbit polyclonal anti-DMD (dilution 1:1000; Sigma-Aldrich). The slides were incubated for 20 min with EnVision FLEX/HRP (DAKO, Glostrup, Denmark). 3,3'-diaminobenzidine (DAB) was used as the peroxidase substrate and the sections were incubated for $10 \mathrm{~min}$. Finally, all sections were counterstained for $5 \mathrm{~min}$ with EnVision FLEX Hematoxylin (Dako). Specimens from uterus, gastrointestinal tract, urinary bladder and skeletal muscles served as positive controls for the expression of smooth muscle and skeletal muscle markers; sections immunostained in the absence of a primary antibody were used as negative controls.

Photomicrographs of the examined tissues were subjected to computer-assisted image analysis, using a computer coupled to an optical Olympus BX53 microscope, equipped with an Olympus Color View IIIu digital camera (Olympus, Tokyo, Japan) and Cell^ ^ A software (Olympus Soft Imaging Solution GmbH, Berlin, Germany).

To allow a semi-quantitative comparison of the examined markers and their correlation with myocardial remodeling, their expression in cardiomyocyte was appraised in 10 randomly chosen high power fields (total magnification $\times 400$ ) using a two-step approach: 1) the intensity of reaction (using the following scoring system: $0-$ no reaction; 1 mild reaction; 2 - moderate reaction; 3 - strong reaction), 2) the percentage of marker-positive cells within a high power field $(\times 400)$. An average score from 10 examined fields was counted for each feature.

Statistical analysis. The normality of the data was evaluated using Shapiro-Wilk analysis. The obtained data underwent statistical analysis using the Kruskal-Wallis rang analysis and Spearman's correlation test using StatisticaPL for Windows (StatSoft, Cracow, Poland). Statistical significance was set at $\mathrm{p} \leq 0.05$.

\section{Results}

The population study of examined dogs is presented in Table 1. The DCM group consisted of eight Doberman Pinchers, four German Shepherds, one boxer, one Great Dane, and one Caucasian Shepherd Dog. The MMVD group included 23 mixed-breed dogs, six dachshunds, two miniature Pinchers and one dog of each breed: Black Russian Terrier, Shar-Pei, Cairn Terrier and the American Staffordshire Terrier. The control group consisted of three mixed-breed dogs and one dog of each breed: Dachshund, Boxer, Yorkshire Terrier.

There was no significant difference between the LA/Ao ratio in DCM and MMVD groups in either the echocardiographic or post-mortem examination $(\mathrm{p}>0.05)$. 
Table 1. Population study of the examined dogs

\begin{tabular}{|l|l|c|c|c|}
\hline \multicolumn{2}{|l|}{} & DCM group $(\mathbf{n}=\mathbf{1 5})$ & MMVD group $(\mathbf{n}=\mathbf{3 5})$ & Control group (n=6) \\
\hline \multirow{3}{*}{ Sex $(\mathrm{n})$} & $\mathrm{M}$ & 13 & 24 & 3 \\
\cline { 2 - 5 } & $\mathrm{F}$ & 2 & 11 & 3 \\
\hline \multirow{3}{*}{ Age (years) } & Mean \pm SD & $8.37 \pm 2.39$ & $13.14 \pm 2.96$ & $7.63 \pm 4.6$ \\
\cline { 2 - 5 } & Min-max & $4-11$ & $8-19$ & $1-12$ \\
\hline \multirow{2}{*}{ Body mass $[\mathrm{kg}]$} & Mean \pm SD & $35 \pm 6.43$ & $13.17 \pm 7.77$ & $1.05 \pm 10.78$ \\
\cline { 2 - 5 } & Min-max & $31-45$ & $5-35$ & $1.03 \pm 0.23$ \\
\hline \multirow{2}{*}{ LA/Ao } & Mean \pm SD & $3.14 \pm 0.33$ & $2.76 \pm 0.65$ & \\
\hline
\end{tabular}

DCM group — dogs with dilated cardiomyopathy; MMVD group — dogs with myxomatous mitral valve disease; M - male; F - female; $\mathrm{SD}$ - standard deviation; $\min$ - minimal value; max — maximal value; LA/Ao — left atrial-to-aortic root ratio.

Table 2. Histopathological characteristics of the studied groups of dogs

\begin{tabular}{|l|c|c|c|}
\hline & DCM group $(\mathbf{n}=\mathbf{1 5})$ & MMVD group $(\mathbf{n}=\mathbf{3 5})$ & Control group (n = 6) \\
\hline Interstitial fibrosis (median; range) & $4(2-7)$ & $2(0-6)$ & $0(0-1)$ \\
\hline Perivascular fibrosis (median; range) & $1(0-2)$ & $2(1-2)$ & $0(0-1)$ \\
\hline Fatty infiltration (median; range) & $2(0-10)$ & $1(0-6)$ & $0(0-2)$ \\
\hline LAR (median; range) & $0.35(0.13-0.61)$ & $0.28(0.13-0.45)$ & $0.32(0.15-0.35)$ \\
\hline Abnormal cell nuclei (median; range) & $2(1-3)$ & $1(0-3)$ & $0(0-1)$ \\
\hline Loss of striation (median; range) & $3(1-3)$ & $1(0-3)$ & $0(0-1)$ \\
\hline Altered cardiomyocyte structure (median; range) & $3(2-3)$ & $1(0-3)$ & $0(0-1)$ \\
\hline Inflammatory infiltration (median; range) & $0.6(0-1.6)$ & $0.33(0-2.1)$ & $0(0-1.6)$ \\
\hline
\end{tabular}

DCM group — dogs with dilated cardiomyopathy; MMVD group — dogs with myxomatous mitral valve disease; LAR — lumen area ratio: the luminal area of the vessel divided by the total vessel area excluding adventitia.

Table 3. The percentages of protein-immunoreactive cells in the examined groups of dogs

\begin{tabular}{|l|c|c|c|}
\hline & DCM group $(\mathbf{n}=\mathbf{1 5})$ & MMVD group $(\mathbf{n}=\mathbf{3 5})$ & Control group $(\mathbf{n}=\mathbf{6})$ \\
\hline SERCA1 (\%) (mean \pm SEM) & $35.01 \pm 8.46$ & $35.61 \pm 5.08^{*}$ & $14.45 \pm 14.45$ \\
\hline SERCA2 (\%) (mean \pm SEM) & $59.51 \pm 10.62$ & $80.77 \pm 3.96$ & $88.75 \pm 3.41$ \\
\hline SA (\%) (mean \pm SEM) & $41.61 \pm 8.87$ & $42.77 \pm 5.33$ & $59.8 \pm 20.6$ \\
\hline SMA (\%) (mean \pm SEM) & $36.1 \pm 9.2$ & $11.73 \pm 2.17$ & $3.63 \pm 0.91$ \\
\hline DMD (\%) (mean \pm SEM) & $19,18 \pm 7.06^{*}$ & $40.36 \pm 6.52$ & $69.57 \pm 11.52$ \\
\hline
\end{tabular}

DCM group — dogs with dilated cardiomyopathy; MMVD group — dogs with myxomatous mitral valve disease; SERCA1 — sarcoplasmic reticulum $\mathrm{Ca}^{2+}$ ATPase 2A-1; SERCA2 - sarcoplasmic reticulum $\mathrm{Ca}^{2+}$ ATPase 2A-2; SA — sarcomeric alpha-actinin; SMA — alpha-smooth muscle actin; DMD — dystrophin; SEM — standard error of mean. *Significantly different from control.

\section{Histopathological examination}

The results of histopathological examination are presented in Table 2. Specimens from the control group showed no or slight histopathological changes. LA tissue from dogs in the DCM and MMVD groups showed signs of remodeling (including interstitial and perivascular fibrosis, fatty infiltration and features of cardiomyocyte degeneration). Moreover, specimens in the DCM group showed lower perivascular fibrosis score, higher interstitial fibrosis score and higher values of cardiomyocyte degeneration features (abnormal cell nuclei, loss of striation and altered cardiomyocyte structure) as compared to specimens from the MMVD group. 


\section{Immunohistochemical examination}

The summary of the results of the IHC investigations is shown in Table 3 and the examples of the immunoreactivity of the studied proteins are presented in Figures 1-5.

\section{SERCA1 and SERCA2}

The expression of SERCA1 protein showed a cytoplasmic pattern in $67 \%$ of specimens in the DCM group and $74 \%$ of specimens in the MMVD group. Two specimens (33.3\%) from a control group showed a positive expression of this protein.

There were no significant differences in the intensity of expression of SERCA1-immunopostive cells in the examined groups $(p>0.05)$. There was a significantly higher percentage of SERCA1-positive cells in the MMVD group as compared to the control group ( $p=0.05$; Tab. 3; Fig. 1). No correlation between SERCA1 immunoreactivity and histological evidence of myocardial remodeling was found $(p>0.05)$.

The expression of SERCA2 protein showed a cytoplasmic pattern in all specimens in control group, $67 \%$ of specimens in the DCM group, and 94\% of specimens in the MMVD group. There were no significant differences in the intensity of immunoreactivity and percentage of SERCA2-positive cells in the examined groups ( $p>0.05$; Tab. 3; Fig. 2).

There was a weak negative correlation between the percentage of SERCA2-positive cells and cardiomyocyte nuclei enlargement $(\mathrm{p}<0.05 ; \mathrm{r}=-0.33)$ and cardiomyocyte degeneration $(\mathrm{p}<0.05 ; \mathrm{r}=-0.29)$. We found no correlation between SERCA2 immunoreactivity and other histological evidence of myocardial remodeling $(\mathrm{p}>0.05)$.

\section{Sarcomeric actinin and SMA}

The expression of SA showed a cytoplasmic pattern in $67 \%$ of specimens in the control group, $73 \%$ of specimens in the DCM group, and $83 \%$ of specimens in the MMVD group. There were no significant differences in the intensity of expression and percentage of striated muscle actin-immunoreactive cells between the examined groups ( $p>0.05$; Tab. 3; Fig. 3). We found no correlation between striated muscle actin expression and histological evidence of myocardial remodeling ( $p>0.05)$.

The immunoexpression of the SMA protein showed a cytoplasmic pattern in less than $6 \%$ of cardiomyocytes in all specimens in the control group, a cytoplasmic pattern of various extents in $80 \%$ of specimens in the DCM group, and $89 \%$ of specimens in the MMVD group. There was no significant difference in the intensity of expression of this protein or percentage of SMA-positive cells in all groups $(p>0.05$; Tab. 3; Fig. 4).

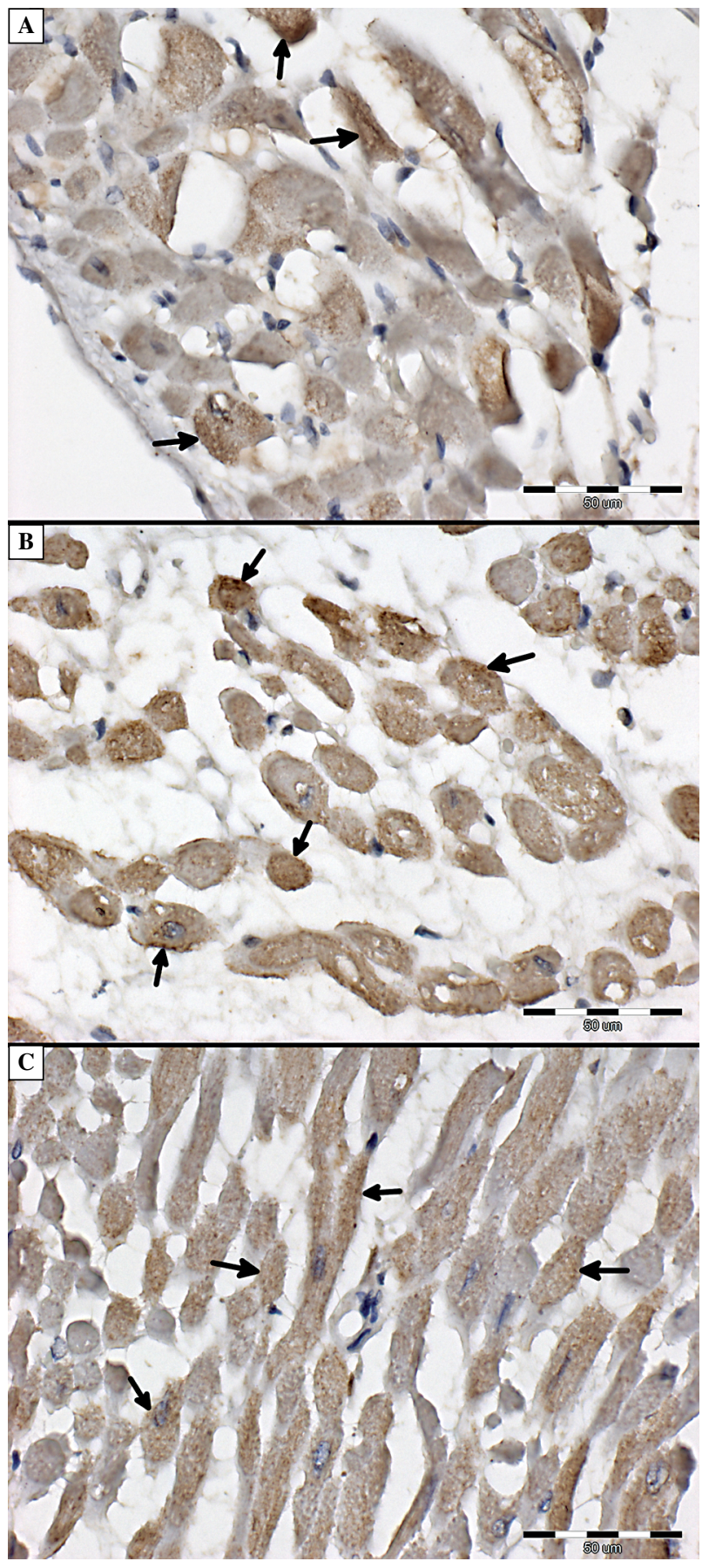

Figure 1. The expression of sarcoplasmic reticulum $\mathrm{Ca}^{2+}$ ATPase 2A-1 (SERCA1) in heart's atria of the examined groups of dogs. The immunoreactivity (IR) of SERCA1 was present in cardiomyocytes' cytoplasm (arrows). A. MMVD group, IR scored 1; B. DCM group, IR scored 1; C. Control group, IR scored 1. Abbreviations as in the description of Table 1 . Magnification $\times 400$.

There was a weak positive correlation of percentage of SMA-positive cells with cardiomyocyte nuclei enlargement ( $\mathrm{p}<0.05 ; \mathrm{r}=0.3)$ and cardiomyocyte degeneration $(p<0.05 ; r=0.27)$. We found no cor- 


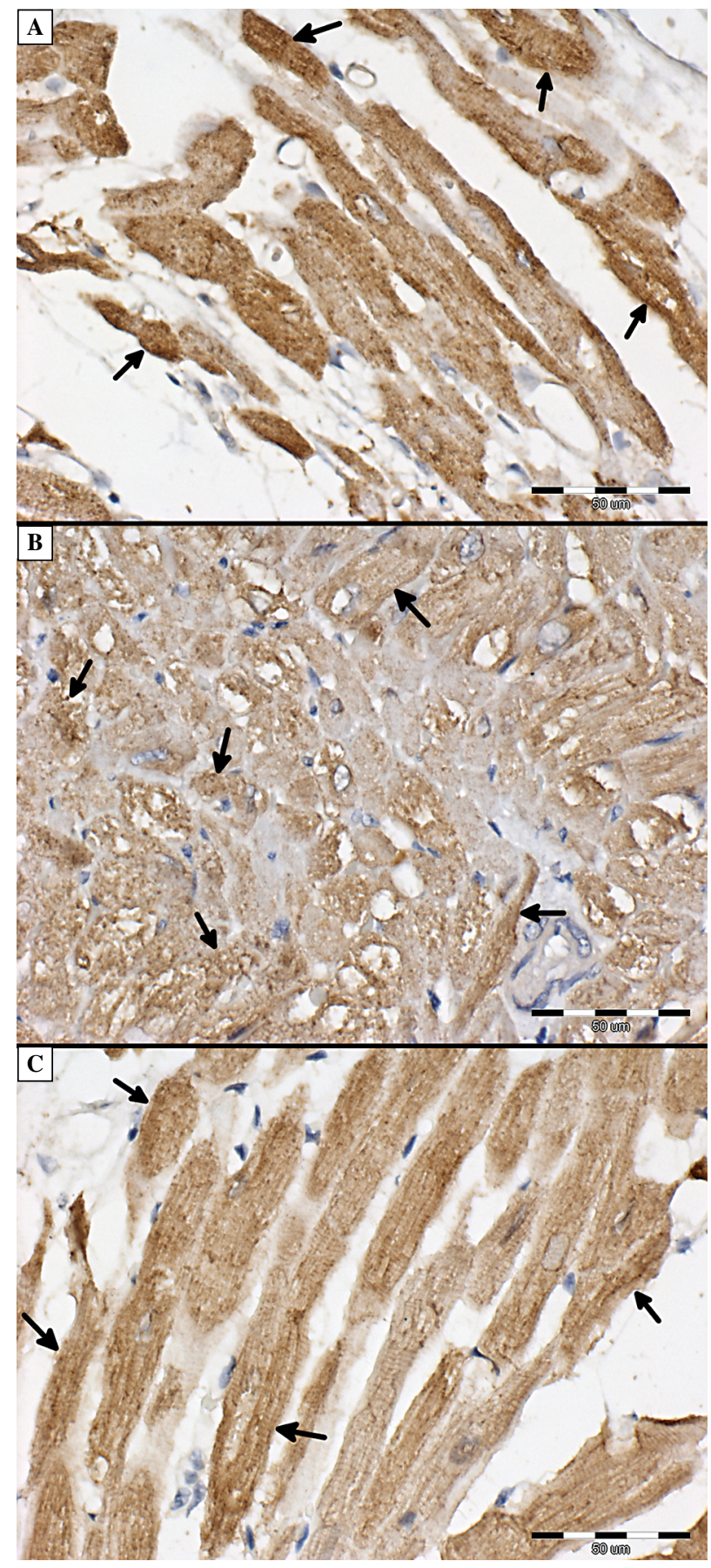

Figure 2. The expression of sarcoplasmic reticulum $\mathrm{Ca}^{2+}$ ATPase 2A-2 (SERCA2) in heart's atria of the examined groups of dogs. The IR of SERCA1 was present in cardiomyocytes' cytoplasm (arrows). A. MMVD group, cardiomyocytes are swollen, IR scored 2; B. DCM group, cardiomyocytes show disarrangement and irregular SERCA2 reactivity, IR scored 1; C. Control group, IR scored 2. Abbreviations as in the description of Figure 1. Magnification $\times 400$.

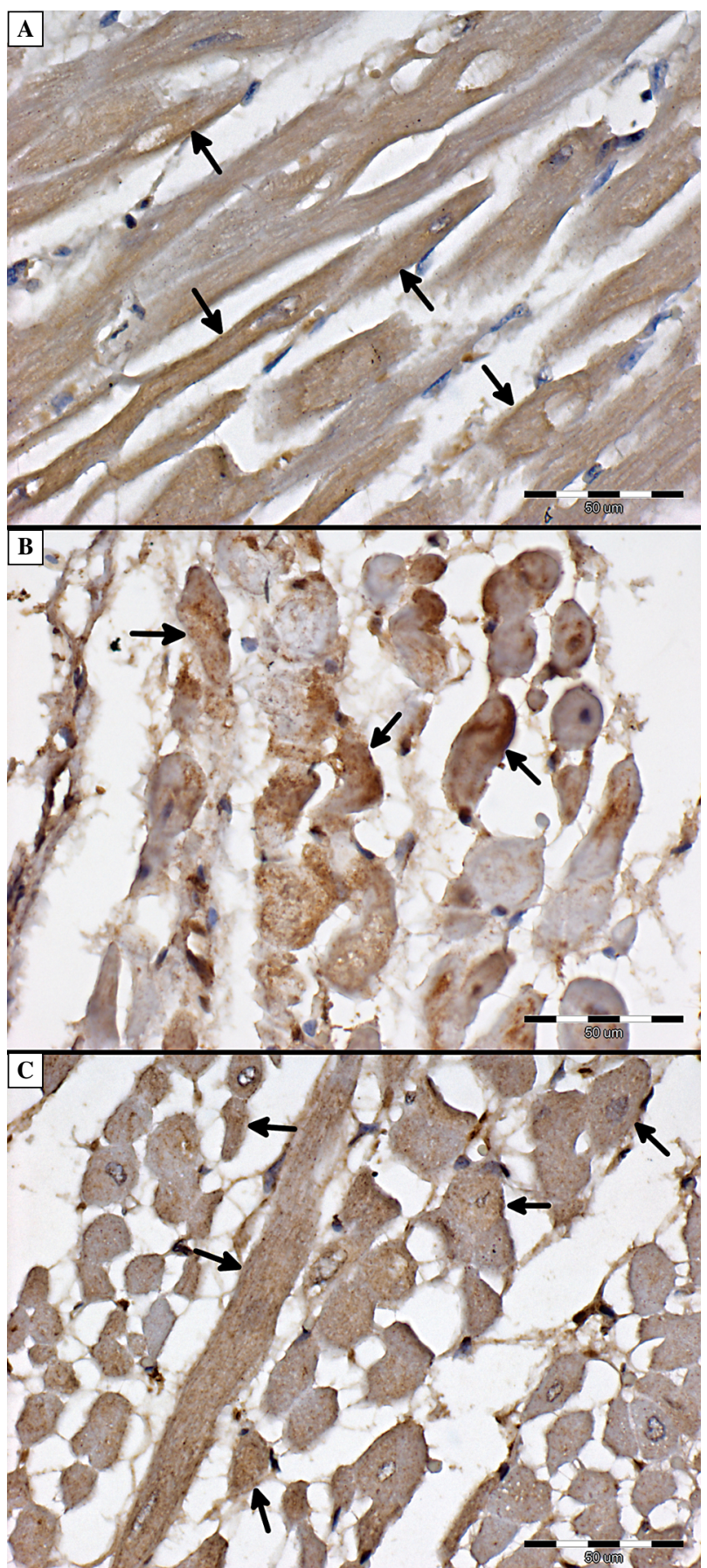

Figure 3. The expression of sarcomeric alpha-actinin (SA) in heart's atria of the examined groups of dogs. The IR of SA was present in cardiomyocytes' cytoplasm (arrows). A. MMVD group, IR scored 1; B. DCM group, IR scored 2; C. Control group, IR scored 1. Abbreviations as in the description of Figure 1. Magnification $\times 400$. 


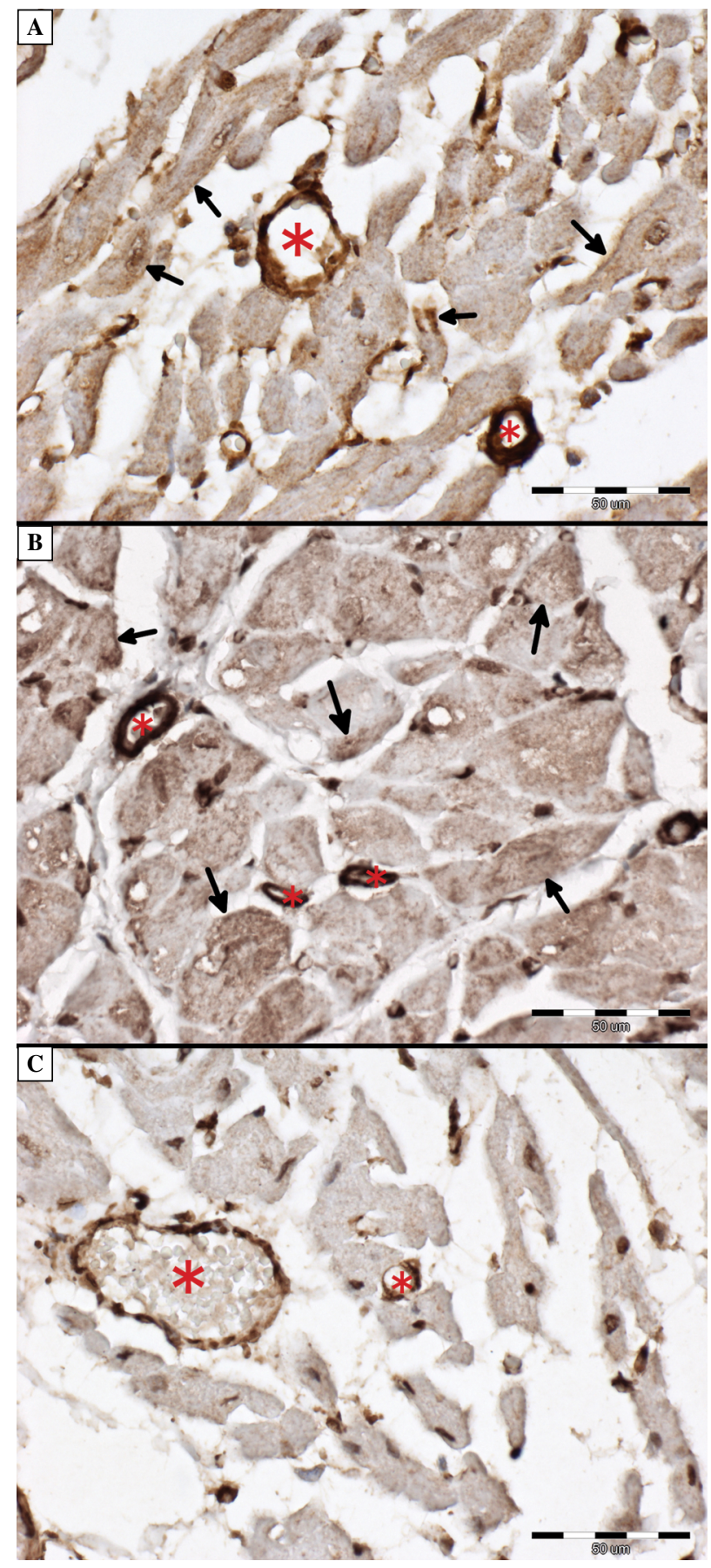

Figure 4. The expression of alpha-smooth muscle actin (SMA) in heart's atria of the examined groups of dogs. The IR of SMA was present in cardiomyocytes' cytoplasm (arrows). Asterisks (*) show blood vessels with strong positive expression of the SMA (internal positive control). A. MMVD group, irregular diffuse expression of SMA protein, IR scored 1; B. DCM group, irregular diffuse expression of SMA, IR scored 1; C. Control group, clear and visible expression of SMA in blood vessels $(*)$ with cardiomyocyte negative for the examined protein. IR scored 1. Abbreviations as in the description of Figure 1. Magnification $\times 400$.
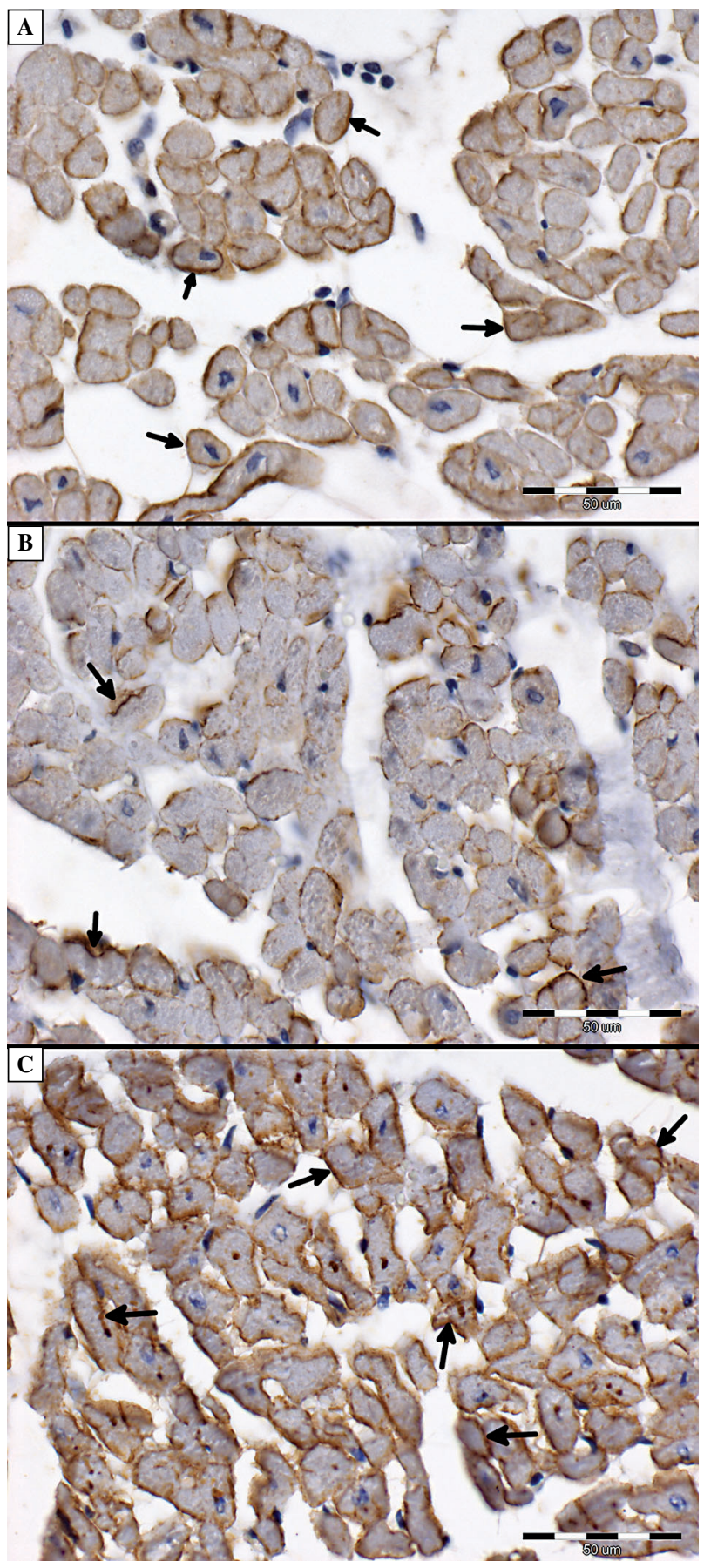

Figure 5. The expression of dystrophin (DMD) in heart's atria of the examined groups of dogs. The IR of dystrophin was present in cardiomyocytes' cell membranes (arrows). A. MMVD group, IR scored 1; B. DCM group, IR scored 1; C. Control group, IR scored 2. Abbreviations as in the description of Figure 1 . Magnification $\times 400$. 
relation between SMA reaction and other histological evidence of myocardial remodeling $(\mathrm{p}>0.05)$.

\section{Dystrophin}

Dystrophin immunoreactivity showed a membranous pattern in all specimens in the control group, in $40 \%$ of specimens in the DCM group, and in 57\% of specimens in the MMVD group. The intensity of the immunoreactivity of dystrophin was significantly higher in the control group as compared to the DCM group $(p=0.02)$. The percentage of DMD-immunopositive cells was significantly lower in the DCM group, as compared to control group ( $\mathrm{p}=0.02$; Tab. 3; Fig. 5).

There was a mild negative correlation between the percentage of DMD-positive cells and features of myocardial damage: cardiomyocyte nuclei enlargement $(\mathrm{p}<0.05 ; \mathrm{r}=-0.42)$, cardiomyocyte degeneration $(\mathrm{p}<0.05 ; \mathrm{r}=-0.43)$, and loss of cardiomyocyte striation $(\mathrm{p}<0.05 ; \mathrm{r}=-0.38)$. We found no correlation between dystrophin immunoreactivity and other histological evidence of myocardial remodeling $(p>0.05)$.

\section{Discussion}

The expression of various cardiomyocyte proteins in left atria of dogs is altered in heart failure, as was noted in this study and previous studies [6,11-13]. Although the majority of studies on SERCA2 expression in heart failure point to a decrease in the protein level, in some studies, the expression levels of SERCA2 was found to be unaltered, similarly to our results $[14,16]$. Regardless the unchanged level of SERCA2 expression, sarcoplasmic reticulum $\mathrm{Ca}^{2+}$ transport function may be decreased [14]. As there is a considerable heterogeneity in the expression level of SERCA in the failing heart, the differences between the obtained results can be explained by a variety of factors, including animal model studied, methodological differences, severity of the disease, treatment, age and gender [14]. It is also possible, that the changes in $\mathrm{Ca}^{2+}$ transport occurring in the ventricles during heart failure are not so prominent in the atria, despite the higher level of SERCA protein in normal atrial myocardium as compared to the ventricles [14].

We found no significant differences in the SERCA2 immunoreactivity between the examined groups; however, there was a higher percentage of SERCA1-positive cells in the MMVD group as compared to the control group. As mentioned before, SERCA1 may undergo expression in myocardium as a part of a natural adaptive process in the failing heart $[15,16]$. Our results are partially consistent with the results obtained by Summerfield et al. [16] who noticed a higher expression of SERCA1 protein in German
Shepherd dogs with DCM as compared to normal canine myocardium. Lack of significant difference between the DCM and control groups in our study may be due to the heterogeneity of the DCM group (apart from German Shepherds, also Dobermans, Boxer dog and Caucasian Shepherd were included). In the aforementioned study [16], authors noted also that the expression of SERCA1 protein was heterogeneous and varied regionally in the specimens obtained from right ventricle, left ventricle and interventricular septum. However, they did not examine specimens from atrial walls, what was the subject of our study.

Smooth muscle proteins, including smooth muscle alpha-actin, show higher content in specimens from human patients with DCM as compared to normal hearts [19]. We found no significant differences between the examined groups in the percentage of SMA-positive cells, although we observed a trend of higher SMA-positive cells percentage in the DCM and MMVD groups, as compared to the control group. Interestingly, in all of the specimens from the control group, less than $6 \%$ of cardiomyocyte showed positive reaction to SMA.

We did not notice differences between the percentage of sarcomeric actinin-positive cells between the control group and the DCM and MMVD groups, although Driesen et al. [17] reported a re-expression of alpha-skeletal muscle actin with a down-regulation of alpha-cardiac actin in various types of heart diseases. As the antibody used in our study did not allow us to make recognition between alpha-cardiac actin and alpha-skeletal muscle actin, we cannot confirm whether such transformation is present in dogs with end-stage DCM and MMVD. An over-regulation of alpha-skeletal muscle actin could disguise a down-regulation of alpha-cardiac actin in the examined material. This issue should be a topic of further investigation.

Dystrophin is a membrane-associated protein that plays a role in preserving the cardiomyocyte contractile function $[8,20]$. Its expression is down-regulated in various diseases, including dystrophinopathies and ischemic heart disease [8, 20-24]. In contrary to the results obtained by Spier et al. [28], we noted a lower staining intensity and percentage of DMD-immunoreactive cells in the DCM dogs as compared to control group. Although the dogs in our study did not undergo genetic examination, none of the dogs in the DCM group presented with progressing skeletal muscle weakness. That allows us to exclude inherited dystrophinopathy as a direct cause of DCM and observed down-regulation of DMD expression in the examined specimens. Although there was no significant difference between the MMVD and control groups in dystrophin immunoreactivity, a visible 
trend of concomitant down-regulation of dystrophin also in the MMVD group and a negative correlation between dystrophin reactivity and features of cardiomyocyte damage were observed. It may suggest that in both DCM and MMVD, atrial tissue is exposed to various stress factors, including ischemia, which can cause loss of dystrophin in cardiomyocytes [23-24]. Nonetheless, a significance noted only in the DCM group as compared to control dogs suggests that in this disease decreased DMD immunoexpression may be connected not only to cardiac remodeling due to volume overload, but also to other pathomechanisms including protein alterations.

\section{Conclusions}

Dilated cardiomyopathy and myxomatous mitral valve disease are both accompanied by left atrial enlargement. In our study on dogs we have noted that some changes occurring in the immunoexpression of proteins building atrial tissue in both diseases are similar; however, there are also significant differences. These findings suggest that other mechanisms, except for volume overload, may be involved in left atrial enlargement in those two diseases. Understanding the mechanisms underlying those diseases may help in the treatment and extend life span of the affected dogs.

\section{Ethical approval}

According to the Polish law [29], standard diagnostic procedures and studies conducted on animal tissue do not require permission from the Ethical Board. Oral consent for the clinical examination was obtained from all owners, as those procedures are used routinely to diagnose canine heart disease.

\section{Acknowledgements}

We would like to thank the supporting staff of the Pathology Department of Wroclaw University of Environmental and Life Sciences and of the Department of Animal Sciences of University of Turin for their experienced technical assistance. The study was supported by Wroclaw Centre of Biotechnology, The Leading National Research Centre (KNOW) program for 2014-2018.

\section{References}

1. Borgarelli M, Savarino P, Crosara S, et al. Survival characteristics and prognostic variables of dogs with mitral regurgitation attributable to myxomatous valve disease. J Vet Intern Med. 2008; 22(1): 120-128, doi: 10.1111/j.1939-1676.2007.0008.x, indexed in Pubmed: 18289298.

2. Dukes-McEwan J, Borgarelli M, Tidholm A, et al. ESVC Taskforce for Canine Dilated Cardiomyopathy. Proposed guidelines for the diagnosis of canine idiopathic dilated cardiomyopathy. J Vet Cardiol. 2003; 5(2): 7-19, doi: 10.1016/ S1760-2734(06)70047-9, indexed in Pubmed: 19081360.

3. Häggström J, Duelund Pedersen H, Kvart C. New insights into degenerative mitral valve disease in dogs. Vet Clin North Am Small Anim Pract. 2004; 34(5): 1209-26, vii, doi: 10.1016/j. cvsm.2004.05.002, indexed in Pubmed: 15325478.

4. Tidholm A, Häggström J, Borgarelli $\mathrm{M}$, et al. Canine idiopathic dilated cardiomyopathy. Part I: Aetiology, clinical characteristics, epidemiology and pathology. Vet J. 2001; 162(2): 92-107, doi: 10.1053/tvj1.2001.0571, indexed in Pubmed: 11531394.

5. Fox PR. Pathology of myxomatous mitral valve disease in the dog. J Vet Cardiol. 2012; 14(1): 103-126, doi: 10.1016/j. jvc.2012.02.001, indexed in Pubmed: 22386587.

6. Hanna N, Cardin S, Leung TK, et al. Differences in atrial versus ventricular remodeling in dogs with ventricular tachypacing-induced congestive heart failure. Cardiovasc Res. 2004; 63(2): 236-244, doi: 10.1016/j.cardiores.2004.03.026, indexed in Pubmed: 15249181.

7. Janus I, Nowak M, Madej J. Pathomorphological changes of the myocardium in canine dilated cardiomyopathy (DCM). Bull Vet Inst Pulawy. 2015; 59(1): 135-142, doi: 10.1515/ bvip-2015-0020.

8. Hein S, Kostin S, Heling A, et al. The role of the cytoskeleton in heart failure. Cardiovasc Res. 2000; 45(2): 273-278, indexed in Pubmed: 10728347.

9. Schaper J, Kostin S, Hein S, et al. Structural remodelling in heart failure. Exp Clin Cardiol. 2002; 7(2-3): 64-68, indexed in Pubmed: 19649225.

10. Sharov VG, Kostin S, Todor A, et al. Expression of cytoskeletal, linkage and extracellular proteins in failing dog myocardium. Heart Fail Rev. 2005; 10(4): 297-303, doi: 10.1007/ s10741-005-7544-2, indexed in Pubmed: 16583178.

11. Janus I, Kandefer-Gola M, Ciaputa R, et al. The immunohistochemical evaluation of selected markers in the left atrium of dogs with end-stage dilated cardiomyopathy and myxomatous mitral valve disease - a preliminary study. Ir Vet J. 2016; 69: 18, doi: 10.1186/s13620-016-0077-2, indexed in Pubmed: 27980728.

12. Janus I, Noszczyk-Nowak A, Nowak M, et al. A comparison of the histopathologic pattern of the left atrium in canine dilated cardiomyopathy and chronic mitral valve disease. BMC Vet Res. 2016; 12: 3, doi: 10.1186/s12917-015-0626-z, indexed in Pubmed: 26728624.

13. Hanna N, Cardin S, Leung TK, et al. Differences in atrial versus ventricular remodeling in dogs with ventricular tachypacing-induced congestive heart failure. Cardiovasc Res. 2004; 63(2): 236-244, doi: 10.1016/j.cardiores.2004.03.026, indexed in Pubmed: 15249181.

14. Periasamy M, Bhupathy P, Babu GJ. Regulation of sarcoplasmic reticulum $\mathrm{Ca} 2+$ ATPase pump expression and its relevance to cardiac muscle physiology and pathology. Cardiovasc Res. 2008; 77(2): 265-273, doi: 10.1093/cvr/cvm056, indexed in Pubmed: 18006443.

15. Sumbilla C, Cavagna M, Zhong L, et al. Comparison of SERCA1 and SERCA2a expressed in COS-1 cells and cardiac myocytes. Am J Physiol. 1999; 277(6 Pt 2): H2381-H2391, indexed in Pubmed: 10600859.

16. Summerfield N, Peters ME, Hercock CA, et al. Immunohistochemical evidence for expression of fast-twitch type sarco(endo)plasmic reticulum Ca2 + ATPase (SERCA1) in German shepherd dogs with dilated cardiomyopathy myocardium. J Vet Cardiol. 2010; 12(1): 17-23, doi: 10.1016/j.jvc.2009.12.001, indexed in Pubmed: 20188644. 
17. Driesen RB, Verheyen FK, Debie W, et al. Re-expression of alpha skeletal actin as a marker for dedifferentiation in cardiac pathologies. J Cell Mol Med. 2009; 13(5): 896-908, doi: 10.1111/j.15824934.2008.00523.x, indexed in Pubmed: 19538254.

18. Gofflot S, Kischel P, Thielen C, et al. Characterization of an antibody panel for immunohistochemical analysis of canine muscle cells. Vet Immunol Immunopathol. 2008; 125(3-4): 225-233, doi: 10.1016/j.vetimm.2008.05.029, indexed in Pubmed: 18617272.

19. Branishte TA, Dudnakova TV, Dergilev KV, et al. Expression of contractile and cytoskeletal proteins in myocardium of patients with dilated cardiomyopathy. Kardiologiia. 2004; 44(12): 31-36, indexed in Pubmed: 15699919.

20. Kamdar F, Garry DJ. Dystrophin-deficient cardiomyopathy. J Am Coll Cardiol. 2016; 67(21): 2533-2546, doi: 10.1016/j. jacc.2016.02.081, indexed in Pubmed: 27230049.

21. Fine DM, Shin JH, Yue Y, et al. Age-matched comparison reveals early electrocardiography and echocardiography changes in dystrophin-deficient dogs. Neuromuscul Disord. 2011;21(7): 453-461, doi: 10.1016/j.nmd.2011.03.010, indexed in Pubmed: 21570848.

22. Nakamura A. X-Linked dilated cardiomyopathy: a cardiospecific phenotype of dystrophinopathy. Pharmaceuticals (Basel). 2015; 8(2):303-320, doi: 10.3390/ph8020303, indexed in Pubmed: 26066469.

23. Armstrong SC, Latham CA, Shivell CL, et al. Ischemic loss of sarcolemmal dystrophin and spectrin: correlation with myo- cardial injury. J Mol Cell Cardiol. 2001; 33(6): 1165-1179, doi: 10.1006/jmcc.2001.1380, indexed in Pubmed: 11444921.

24. Rodríguez M, Cai WJ, Kostin S, et al. Ischemia depletes dystrophin and inhibits protein synthesis in the canine heart: mechanisms of myocardial ischemic injury. J Mol Cell Cardiol. 2005; 38(5): 723-733, doi: 10.1016/j.yjmcc.2005.02.019, indexed in Pubmed: 15850566.

25. Tidholm A, Jönsson L. Histologic characterization of canine dilated cardiomyopathy. Vet Pathol. 2005; 42(1): 1-8, doi: 10.1354/vp.42-1-1, indexed in Pubmed: 15657266.

26. Whitney JC. Cardiovascular pathology. J Small Anim Pract. 1967; 8(8): 459-465, doi: 10.1111/j.1748-5827.1967.tb04575.x.

27. Atkins C, Bonagura J, Ettinger S, et al. Guidelines for the diagnosis and treatment of canine chronic valvular heart disease. J Vet Intern Med. 2009; 23(6): 1142-1150, doi: 10.1111/j.19391676.2009.0392.x, indexed in Pubmed: 19780929.

28. Spier AW, Meurs KM, Coovert DD, et al. Use of western immunoblot for evaluation of myocardial dystrophin, alpha-sarcoglycan, and beta-dystroglycan in dogs with idiopathic dilated cardiomyopathy. Am J Vet Res. 2001; 62(1): 67-71, doi: 10.2460/ajvr.2001.62.67, indexed in Pubmed: 11197563.

29. The Polish Parliament (Sejm) [Internet]. Warsaw: The Polish Parliament: Act on the protection of animals used for scientific or educational purposes; c2015. http://orka.sejm.gov.pl/ proc7.nsf/ustawy/2709_u.htm. (2017 March 28).

Submitted: 21 March, 2017 Accepted after reviews: 7 June, 2017 Available as AoP: 12 June, 2017 